\title{
FLORA DEL MONUMENTO NATURAL EL MORADO: ADDENDA ET CORRIGENDA
}

\section{FLORA OF THE MONUMENTO NATURAL EL MORADO: ADDENDA ET CORRIGENDA}

\author{
S. Teillier \\ Escuela de Ecología y Paisaje, Universidad Central, Santiago, Chile. E-mail: steillier@chlorischile.cl

\section{RESUMEN} \\ Este artículo tiene como objetivo mejorar el conocimiento de la flora vascular del Monumento Natural El Morado, \\ ubicado cerca de Santiago en Chile central. El total de especies reportadas se aumenta de 280 a 300, de las cuales 263 \\ son nativas y 37 exóticas. \\ Palabras claves: Estepa andina, flora andina, matorral andino, SNASPE
}

\section{ABSTRACT}

This paper is intended to enhance knowledge of the vascular flora of Monumento Natural El Morado, located near Santiago in central Chile. The total of 280 species reported previously is now increased to 300, of which 263 are native and 37 alien.

Keywords: Andean flora, Andean grasslands, Andean shrublands, SNASPE

\section{INTRODUCCION}

El Monumento Natural El Morado es parte del Sistema Nacional de Areas Protegidas del Estado de Chile (SNASPE). Se sitúa en la Región Metropolitana, en la provincia Cordillera, comuna de San José de Maipo. Su flora vascular fue estudiada por Teillier et al. (1994), los que refieren la presencia de 280 especies, 248 nativas y 32 alóctonas.

En el verano del 2001-2002 realizamos una serie de excursiones a esta área protegida. Como resultado de ellas presentamos nuevos registros para su flora vascular. Además, se revisaron las determinaciones realizadas entonces, basándonos en un mayor número de colecciones y en literatura taxonómica nueva. Por último, damos cuenta de una serie de cambios de denominación de especies presentes en el parque como producto de las múltiples revisiones genéricas que se han publicado desde 1994.

El objetivo de esta nota es complementar el trabajo de Teillier et al. (1994) respecto de la flora del M. N. El Morado con nuevos registros, discutir algunas de las determinaciones publicadas en 1994 y hacernos cargo de las modificaciones nomenclaturales que se han propuesto a la fecha.

A continuación la lista de las nuevas especies encontradas y las modificaciones nomenclaturales habidas hasta la fecha. Los materiales citados como "ST" corresponden a colectas del autor citadas en Teillier et al. (1994). 
Flora Monumento Natural El Morado: TeILlier , T.

\section{PTERIDOPHYTA}

Equisetaceae

Equisetum bogotense Kunth, Humboldt, Bonpland et Kunth, Nov. Gen. Sp. (ed.qu.) 1:42. 1816.

Hierba perenne, rizomatosa. Tallos de 10-20 cm altura. Rizoma subterráneo, bien ramificado. Tallos aéreos delgados, 4-6 valéculas profundas. Hojas de 3-6 mm, soldadas entre sí. Estróbilos terminales, de 1,5-2,5 cm, sobre pedúnculos de 1-1,5 $\mathrm{cm}$.

Sólo se encontró en una vega del cerro Ruhillas (2000 m). Corresponde a un registro nuevo para el área de estudio. Teillier y Márquez 5172 (CONC).

\section{PINOPHYTA}

\section{EPHEDRACEAE}

Ephedra chilensis K. Presl, Abh. Koenigl

Boehm. Ges. Wiss., ser. 5,3: 431. 1845

En Teillier et al. (1994) está citada como Ephedra andina Poepp. ex C. A. Mey.

\section{MAGNOLIOPSIDA (MAGNOLIOPHYTA)}

\section{Campanulaceae}

Lobelia oligophylla (Wedd.) T.G.Lammers, Novon 9 (1): 74.1999

En Teillier et al. (1994), como Hypsela reniformis (Kunth) K. Presl.

\section{Chenopodiaceae}

Chenopodium chilense Schrad., Index Sem. Hort. Gott. 2.1832

Hierba perenne. Follaje aromático. Hojas, alternas, glandulosas, en su mayoría angostas, laciniado aserradas, si ovado-elípticas, entonces sinuadolobuladas, de $2-10 \mathrm{~cm}$. Inflorescencias casi carentes de brácteas dispuestas en espigas densas y ramificadas, $2-5 \mathrm{~cm}$ largo. Cáliz fructífero mo- dioliforme, de 1,4 a 1,6 mm diámetro. Flores con perigonio glanduloso, pubescente. Semillas horizontales y verticales, negro-brillantes.

Frecuente sólo en el sector de la entrada por Baños Morales, en los alrededores del refugio de CONAF. 1800-1850 m. Nuevo registro para el área. Teillier y Márquez 5171 (CONC).

\section{Compositae}

Chaetanthera moenchioides Less., Syn. Comp. 113. 1832.

Hierba anual, de $4 \mathrm{~cm}$. Tallos horizontales. Hojas opuestas, agrupadas en la base de la planta, seríceopubescentes, lineal-subuladas, de 9-15 x 0,5-2 mm, ápice tridentado. Cabezuelas solitarias, involucro acampanado de $6 \mathrm{~mm}$ diámetro; flores bilabiadas, las externas blancas, las internas, amarillas.

Poco frecuente, crece en los alrededores de la entrada al área de estudio. 1800-1850 m. Nuevo registro para el área. Teillier y Márquez 5213 (CONC).

Gnaphalium andicola Phil., Anales Univ. Chil. 90:17. 1895

Hemos confirmado la identificación del ejemplar Teillier 2301 como perteneciente a esta especie al que agregamos Teillier y Márquez 5315 (CONC). La especie ha sido incluida en la sinonimia de Gnaphalium cheiranthifolium, una especie del litoral, de la que se diferencia por ser de talla mucho menor y por tener las hojas más estrechas.

Gnaphalium gayanum Remy, in Gay, Fl. Chil. 4: 225.1847

Hierba perenne, de hasta $25 \mathrm{~cm}$. Hojas verdosas, atenuadas en la base, con el margen suavemente crenado; de 3-6 x 0,5 cm. Cabezuelas terminales, brácteas ovado-elípticas, de color pardo o crema. Es una especie similar a Gnaphalium andicola, pero difiere por tener las brácteas del involucro de un color diferente y por los márgenes de las hojas que no son enteros.

Anderberg (1989) propuso su traspaso a Pseudognaphalium gayanum (Remy) A. A. Anderb.

El tipo de la especie es del valle del río 
Yeso, también tributario andino del río Maipo, situado al NW del área de estudio.

Escaso en el área de estudio. Teillier y Márquez 5313 (CONC), 5314 (CONC).

Haplopappus arbutoides Remy, in Gay, Fl. Chil. 4:53. 1847

Los ejemplares referidos a esta especie deben referirse a Haplopappus velutinus Remy.

Haplopappus illinitus Phil., Linnaea 28:727. 1858

Hall (1928) indica que se trata de una especie muy relacionada con Haplopappus velutinus Remy. Tortosa \& Bartoli (2002) la tratan como sinónimo.

Haplopappus velutinus Remy, in Gay, Fl. Chile 4: 57. 1847

Subarbusto de tamaño muy variable, tallos ascendentes rematados por una cabezuela solitaria. Hojas desde velutinas hasta glutinosas, pecioladas, las basales con pecíolo tan largo como la lámina, elípticas, dentadas, de $1-3,5$ (4) cm. Cabezuelas radiadas, involucro de $1-1,5 \mathrm{~cm}$ altura. Brácteas dispuestas en tres series, lineares, mucronadas, de menos de $1 \mathrm{~mm}$ de ancho. Aquenios villosos.

A esta especie deben referirse los materiales citados por Teillier et al. (1994) bajo los números: 2358, 2359, 2365-b (Haplopappus arbutoides) y 2365-a (Haplopappus illinitus).

Lactuca virosa L., Sp. Pl. 795. 1753

A esta especie debe referirse el material citado por Teillier et al. (1994) como Lactuca serriola L.

Planta cuya presencia en Chile fue recientemente registrada por Macaya et al. (1999).

Leucheria bridgesii Hook. et Arn., in Hooker, Comp. Bot. Mag. 1:36. 1835

Hierba perenne de hasta $35 \mathrm{~cm}$ altura. Tallos glandulosolanosos, ramificados desde la base. Hojas inferiores pecioladas, con lana blanca, especialmente en el envés, elípticas, de 5 x 1-1,5 cm. Hojas caulinares lanceoladas a oblanceoladas, $1-6$ x 0,3-1 cm. Cabezuelas con involucro de 4-6 mm diámetro x 4-5 mm de altura; pocas flores entre las brácteas internas (páleas). Flores 4,5-6 mm.
Crece en el matorral andino. 2000-2300 m. Teillier y Márquez 5307-A (CONC).

Mutisia acerosa Poepp. ex Less., Syn. Comp. 107. 1832

Arbusto de hasta $40 \mathrm{~cm}$ altura. Tallos glabros. Hojas sésiles, subuladas, punzantes, de hasta $4 \mathrm{~cm}$ longitud. Cabezuelas cortamente pedunculadas, involucro de 15 $\mathrm{mm}$. Flores blancas, bilabiadas, dimorfas: las externas, blancas en la haz y rosadas en el envés, las internas, amarillas.

Crece en el matorral andino de la parte inferior. 1800-2000 m. Teillier y Márquez 5178 (CONC), 5179 (CONC).

\section{Crassulaceae}

Crassula connata (Ruiz et Pav.) A.Berger, in Engl. \& Prantl, Nat. Pflanzenfam. ed. 2, 18 (a) 389. 1930.

Hierba diminuta. Hojas de hasta $7 \mathrm{~mm}$, lineares. Flores sobre pedicelos de hasta $10 \mathrm{~mm}$; tetrámeras, carpelos uniseminados. Semilla lustrosa con estrías longitudinales y glándulas pequeñas.

Escasa. Se la encontró sólo en los alrededores de la laguna, en una depresión donde se acumula nieve, crecía junto con Limosellaaustralis y Juncus bufonius. 2400 m. Teillier y Márquez 5176 ( CONC).

\section{Cruciferae}

Camelina alyssum (Mill.)Thell., in Verz. Sam. u. Fruchte Bot. Gart. Zurich, 1906, 10; Bull. Herb. Boiss. Ser.2. (7):183.1907.

Hierba anual, de hasta $40 \mathrm{~cm}$ altura. Hojas lineares de hasta $2,5 \mathrm{~cm}$. Flores dispuestas en racimos ramificados, pedicelos maduros de $7 \mathrm{~mm}$. Flores desconocidas. Fruto una silícula de 5 x 2,5 mm.

Poco frecuente, se la encontró en la parte inferior. 1900-2000 m. Planta alóctona, asilvestrada. Nueva para el área de estudio. Teillier y Márquez 5186 (CONC, sólo material con frutos).

Ramírez y Escaff (1968) registran esta especie para Chile por primera vez, pero no citan material. Marticorena y Quezada (1985), a su vez, no la incluyen en su catálogo. Este es el primer registro para la especie respaldado por material de herbario. 
Flora Monumento Natural El Morado: TeILlier, S.

Menonvillea scapigera (Phil.) Rollins, Contr. Gray Herb. 117: 32.1955

De acuerdo con Prina (2001), a este taxón debe referirse el material citado por Teillier et al. (1994) como Menonvillea hookerii Rollins.

Rorippa bonariensis (Poir.) Makloskie, Rep. Princeton Univ. Exped. Patagonia 8, Bot. 434. 1905

Hierba perenne, de hasta $50 \mathrm{~cm}$ altura. Hojas pinnatisectas, lóbulos lineales, enteros, 4-6 pares. Flores blanco-amarillentas. Silículas glabras de hasta $10 \mathrm{~mm}$ largo, ápice apiculado.

Escasa. Se colectó sólo una vez en un curso de agua pequeño, cerca de la laguna. $2350 \mathrm{~m}$. Corresponde a un nuevo registro para el área del estudio. Teillier y Márquez 5185 (CONC).

\section{LOASACEAE}

Loasa insons Poepp., Fragm. Syn. Pl. Chil. 24. 1833

De acuerdo con Grau (1996) a este taxón pertenece el material citado por Teillier et al. (1994) bajo Loasa tricolor Ker-Gawl. var. insons (Poepp.) Urb. et Gilg.

Loasa paradoxa Urb.\& Gilg, Nova Acta Acad. Caes. Leop.-Carol. German. Nat. Cur. 76: 156. 1900

Se encontró un ejemplar en CONC (Zoellner 2478), colectado en el cerro Ruhillas, que podría incluirse en la flora del área de estudio. La determinación es de J. Grau.

\section{ONAGRACEAE}

Clarkia tenella (Cav.) F.H.Lewis \& M. R. Lewis, Madroño, 12:34. 1953

Hierba anual, erecta, de hasta $30 \mathrm{~cm}$. altura. Hojas alternas, lineares, hasta $15 \mathrm{~mm}$. Flores solitarias en las axilas de las hojas superiores, pétalos morados, de 5-7 mm. Cápsula erecta, paralela al tallo, de $20 \mathrm{~mm}$.

Poco frecuente en la parte inferior del área de estudio. 1800-2000 m. Nuevo registro para el área del estudio. Teillier y Márquez 5194 (CONC).

\section{Plantaginaceae}

Plantago major L. Sp. Pl. 112. 1753

Hierba perenne, rizomatosa. Hojas en roseta, base atenuada a modo de pecíolo, glabras, lámina de 6$8 \times 2,5-3 \mathrm{~cm}$, margen irregularmente dentado, dientes de hasta $1 \mathrm{~mm}$. Espigas de hasta $6 \mathrm{~cm}$, dispuestas sobre pedúnculos de hasta $20 \mathrm{~cm}$ altura. Fruto, una cápsula de 4-6 $\mathrm{mm}$. Semillas negras de $1 \mathrm{~mm}$.

Escasa, crece en las vegas del cerro Ruhillas. Especie alóctona, asilvestrada. Nuevo registro para el área de estudio. Teillier y Márquez 5180 (CONC).

\section{R ANUNCULACEAE}

Caltha sagittata Cav., Ic. 5. 8, 456. t. 414.1799

Hierba perenne, acaule, rizomatosa. Hojas con pecíolos de hasta $5,5 \mathrm{~cm}$, lámina ovada a subtriangular, de $2 \times 2,5 \mathrm{~cm}$, base sagitada, borde irregularmente crenado, apéndices oblicuos, tan largos como la lámina. Flores subsésiles o cortamente pediceladas, Perigonio petaloide. Carpelos libres. Fruto, un folículo.

Escasa. Se encontró en una vega de gran tamaño situada entre la laguna y el glaciar. Constituye un nuevo registro para el área del estudio. Teillier y Márquez 5170 (CONC).

\section{SCROPHULARIACEAE}

Calceolaria arachnoidea Graham, Edinb. N. Phil. J. (Sept. 1828):371. 1828

De acuerdo con Erhart (2000), las plantas del área de estudio corresponden a Calceolaria arachnoidea Graham subesp. nubigena C.Erhart.

Calceolaria corymbosa Ruiz et Pav. subesp. mimuloides (Clos) C.Erhart, Biblioth. Bot. 153: 130

De acuerdo con Erhart (2000), a este taxón debe referirse el material citado en Teillier et al. (1994) como Calceolaria mimuloides Clos. 
Gayana Bot. 60(2), 2003

Calceolaria corymbosa Ruiz et Pav. subesp. santiaguina C.Erhart, Biblioth. Bot., 153: 129

De acuerdo con Erhart (2000), a este taxón debe referirse el material citado en Teillier et al. (1994) como Calceolaria cana Cav.

Calceolaria filicaulis Clos subesp. luxurians (Witasek) C.Erhart, Biblioth. Bot.153: 167. 2000

De acuerdo con Erhart (2000), a este taxón corresponde al material citado por Teillier et al. (1994) como Calceolaria biflora Lam. var. obtusifolia (Kunze ex Walp. et Schauer) Reiche.

Limosella australis R. Br., Prod. 443.1810

Hierba anual, acaule. Hojas en rosetas, lineales de hasta $4 \mathrm{~cm}$. Flores solitarias, axilares, campanuladas, blancas, cáliz y corola pentámeros, con 4 estambres. Fruto, una cápsula glabra, de hasta $2 \mathrm{~mm}$.

Poco frecuente. Se la colectó cerca de la laguna en un sitio inundado. Crece con Juncus bufonius y Crassula connata. Corresponde a un nuevo registro para el área del estudio. Teillier y Márquez 5177 (CONC).

\section{LILIOPSIDA}

\section{AmARYLLIDACEAE}

El material referido a Hippeastrum herbertianum debe referirse a Rhodophiala rhodolirion (Baker) Traub.

\section{Cyperaceae}

Carex decidua Boott, Boott in Proc. Linn. Soc. i. (1845) 255

Hierba perenne, provista con rizomas, de hasta 30 $\mathrm{cm}$. altura. Hojas lineares, graminiformes. Espigas sobre un pedúnculo de hasta $30 \mathrm{~cm}$ altura. Espiguillas pseudolaterales, de hasta 1,5 cm largo; unisexuales excepto la superior con flores masculinas basales y femeninas en la superior. Brácteas violáceas, sólo la nervadura verde. Flores femeninas con dos estigmas.

Poco frecuente. Se colectó sólo en una vega situada entre la laguna y el glaciar. Corresponde a un nuevo registro para el área del estudio. Teillier y Márquez 4946 (CONC).

Eleocharis cf. pachycarpa E.Desv., in C. Gay, Fl. Chil. 6:174. 1854

Hierba perenne, provista de rizomas. Hojas aciculares, de hasta $4 \mathrm{~cm}$, con la base rojiza. Inflorescencias sobre pedúnculos de hasta $5 \mathrm{~cm}$, vaina basal de color pardo claro con borde horizontal, espiguillas de $5-8 \mathrm{~mm}$, bráctea basal carenada en el dorso, aguda, de hasta 5 $\mathrm{mm}$. Flores femeninas con estigma tripartido. Fruto, un utrículo triangular, liso, brillante, de $2 \mathrm{~mm}$.

Referimos a esta especie algunos ejemplares colectados en una vega situada entre la laguna y el glaciar. Difiere notablemente de E. albibracteata por su tamaño y espiguillas mayores. Se trata de una entidad nueva para el área del estudio. Teillier y Márquez 4941 (CONC).

Phylloscirpus acaulis (Phil.) Goetgh. \& D.A. Simpson, Kew Bull., 46 (1): 175. 1991

A esta especie corresponde el material citado por Teillier et al. (1994) como Scirpus acaulis Phil.

GRAMinEAE

Agrostis leptotricha E. Desv. in Gay, Fl. Chil. 6.316. 1854

A este taxón pertenecen los ejemplares citados como Agrostis insconspicua Kunze, una especie muy afín, pero de menor tamaño.

Cynosurus echinatus L., Sp. Pl. 72. 1753

Hierba anual, de hasta $50 \mathrm{~cm}$ altura. Hojas con láminas de hasta $10 \mathrm{~cm}$. Lígula membranácea. Panícula contraída, capitada, de hasta $2 \mathrm{~cm}$. Espiguillas 8-10 $\mathrm{mm}$, con flores fértiles y estériles, aristas largas, hasta $10 \mathrm{~mm}$, exsertas y violáceas.

Poco frecuente, se encontró en el margen de una vega, en la base del cerro Ruhillas. Especie alóctona, asilvestrada. Nuevo registro para el área del estudio. Teillier y Márquez 5182 (CONC).

Poa gayana E.Desv. in C. Gay, Fl. Chil. 6. 416. 1854

Parte de los materiales de Poa depositados en CONC, 
i.e. ST 2538, han sido etiquetados por el Prof. R.J. Soreng asignando el material a esta especie. Al parecer es la especie más abundante en el sector de menor altitud del área de estudio; Poa holciformis es más frecuente sobre $2400 \mathrm{~m}$, pudiendo alcanzar hasta los $3400 \mathrm{~m}$.

Otros ejemplares que podrían ser atribuidos a esta especie serían: Teillier y Márquez 5246 (CONC) y 5247 (CONC). De mantenerse la determinación correspondería a una nueva especie para el área del estudio.

Poa secunda J. \& C.Presl, Rel. Haenk. 1:271.1830

Hierba cespitosa, perenne. Hojas muy estrechas, de hasta $5 \mathrm{~cm}$, lígula hialina, aguda de $5 \mathrm{~mm}$, lámina conduplicada, margen mucronulado. Panícula de contraída, de hasta $5 \mathrm{~cm}$ largo, sobre pedúnculos de hasta $20 \mathrm{~cm}$. Espiguillas sobre pedúnculos de hasta 7 $\mathrm{mm}$; glumas de hasta $2 \mathrm{~mm}$, la superior más ancha y violácea; antecios 2; lemma mútica, $3 \mathrm{~mm}$, con la base ciliada, 5 nervaduras, pálea de $3 \mathrm{~mm}, 2$ nervios y carenaciliada.

Crece en sitios húmedos, especialmente en las cercanías de la laguna de El Morado. 2200-2500 m. Nuevo registro para el área del estudio. Teillier y Márquez 5283 (CONC).

Polypogon monspeliensis (L.) Desf., Fl. Atlant. 1: 67. 1798

Hierba anual. Tallos floríferos de hasta $20 \mathrm{~cm}$. Hojas con láminas de hasta $7 \mathrm{~cm}$, planas. Panícula de hasta $3 \mathrm{~cm}$, muy contraída, espiciforme. Espiguillas con pedúnculo escabro. Glumas similares, lanceoladas, 22,5, ápice brevemente 2-lobulado, arista subapical, 6$8 \mathrm{~mm}$; lemma de 0,9-1 cm, arista de 1-1,5 mm.

Escasa, colectada en el margen de la vega del cerro Ruhillas. Especie alóctona, asilvestrada. Nuevo registro para el área de estudio. Teillier y Márquez 5181 (CONC).

IRIDACEAE

Olsynium philippi (Klatt) Goldblatt, Syst. Bot. 15 (3): 508. 1990

A este taxón debe referirse el material citado por Teillier et al. (1994) como Sisyrinchium philippi Klatt.
Sisyrinchium arenarium Poepp. subesp. adenostemon (Phil.) Ravenna, Bol. Soc. Argent. Bot. $11(2-3): 156.1967$

A este taxón deben referirse los materiales citados en Teillier et al. (1994) como Sisyrinchium arenarium Poepp.

\section{JunCACEAE}

Juncus stipulatus Nees \& Meyen in Nov. Act. Nat. Cur. 19. Suppl. 1:126.1843

De acuerdo con J. Kirschner (com. pers.) a este taxón corresponde el material citado por Teillier $e t$ al. (1994) como Juncus chilensis Gay.

De acuerdo con los datos presentados, la riqueza de la flora del MN El Morado alcanzaría a unos 302 taxa: 300 especies y dos subespecies. En relación con el origen geográfico de las especies, 263 son nativas $(87,6 \%)$ y 37 , alóctonas, asilvestradas $(12,4 \%)$

Arroyo et al. (2000), al analizar comparativamente la flora vascular nativa del M. N. El Morado con la de las otras áreas silvestres protegidas de Chile Central, indican que la riqueza registrada por Teillier et al. (1994), 249 especies, estaba por debajo de los valores predichos por un modelo de potencia, log-log, que relaciona para la flora de Chile central, la superficie con la riqueza. Al respecto, si bien el número de especies registradas, 265, sigue siendo menor al esperado de 273, se observa que es bastante más cercano. En relación con la dificultad para alcanzar los valores de riqueza esperables, existen en el área importantes restricciones ambientales:

1. Un gradiente de altitud que va de 1800 a 3400 $\mathrm{m}$, pero donde la mayor superficie del parque se encuentra entre los 2000 y los $3000 \mathrm{~m}$. Este rango es comparativamente menor que el del S. N. Yerba Loca, que incluye comunidades de plantas que se desarrollan desde los 1500 y los 3800 m. Es importante señalar, además, que las laderas donde la altitud llega hasta $3400 \mathrm{~m}$ presentan pendientes muy altas que dificultan su colonización, además, las partes más altas corresponden generalmente a "filos" y no a mesetas donde la vegetación pueda desarrollarse.

2. La enorme extensión ocupada por los "acarreos", 
es decir, laderas, muy inclinadas que presentan grandes extensiones de coluvios. Estos cubren la mayor parte de las laderas del cajón de Morales. Estos "acarreos", por su gran inestabilidad, son colonizados por muy pocas especies.

3. La escasa superficie del área protegida que presenta suelos bien desarrollados.

De acuerdo con datos de Kong (1997), quien analizó la flora del S. N. Yerba Loca, sobre los 2000 $\mathrm{m}$ de altitud, la similitud, medida como coeficiente de Söhrensen, de ambas floras vasculares, considerando altitudes equivalentes, alcanzaría a un $52,6 \%$. Esto indica que ambas unidades tienen, desde el punto de vista de la conservación de la flora andina de la Región Metropolitana, un carácter de complementarias, lo que fortalece su estatus de áreas andinas protegidas.

El establecimiento de la categoría "Monumento Natural" para esta área protegida se hizo con el objetivo principal de conservar el paisaje, que incluye el glaciar y la laguna de Morales. Los estudios de la flora demuestran que la protección del área resulta, además, del mayor interés para contribuir a la protección de las comunidades vegetales andinas de Chile Central.

\section{AGRADECIMIENTOS}

Este trabajo se realizó con el financiamiento de la Universidad Central de Santiago de Chile. Se agradece a J.Mella, C.Márquez, A.Urquiza y D.Pedraglio por su ayuda en terreno. Por las facilidades para trabajar en los respectivos herbarios el autor agradece a C.Marticorena, A.Marticorena y R.Montero CONC y M.Muñoz e I.Meza SGO. Las determinaciones contaron con la ayuda de G. Wheeler (Carex), Jan Kirschner (Juncus) y Patricio López (Crassula).

\section{BIBLIOGRAFIA}

Anderberg, A.A. 1989. Phylogeny and reclassification of the tribe Inulae (Asteraceae). Canadian Journal of Botany 67: 2277-2296.

Arroyo M. T. K.; O. Matthei; C. Marticorena \& M. Muñoz. 2000. Flora Vascular del Santuario de la Naturaleza Yerba Loca, $33^{\circ}$ S. Región Metropolitana. Informe para CONAF. $45 \mathrm{pp}$.

Erhart, C. 2000. Die Gattung Calceolaria (Scrophulariaceae) in Chile. Bibliotheca Botanica Heft 153. Stuttgart. 283 pp.

Grau, J. 1996. Andean permutations on three closely related Loasa species from Central Chile. In: Annalen des Naturhistorischen Museums in Wien Serie B Botanik und Zoologie. 98 (SUPPL.): 463-476.

Hall, H. M. 1928. The genus Haplopappus. A phylogenetic study in the Compositae. Carnegie Institution of Washington Publication 389: i-viii, 1-391, 16 lám.

Kong, S. 1997. Estudio florístico del Santuario de la Naturaleza Yerba Loca. Región Metropolitana. Chile. Estudio Monográfico. Escuela de Ecología y Paisajismo. Universidad Central de Santiago de Chile. 91 pp.

Macaya, J.; L. Faúndez \& M. T. Serra. 1999. Lactuca virosa $\mathrm{L}$. (Asteraceae), nuevo registro para la flora advena de Chile. Gayana Botanica 56: 141-143.

Marticorena, C \& M. Quezada. 1985. Catálogo de la flora vascular de Chile. Gayana Botanica 42: 1-157.

Prina, A. 2001. Nuevas combinaciones en Menonvillea (Brassicaceae). Hickenia 3 (26): 93-94.

Ramírez, A. \& M. Escaff. 1968. Malezas de Chile. Boletín Técnico . SAG-Chile. 34: 126 (18).

Teillier, S; A. J. Hoffmann; F. Saavedra \& L. Pauchard. 1994. Flora del Parque Nacional El Morado (Región Metropolitana, Chile). Gayana Botanica 51: 13-47.

Tortosa, R. \& A. Bartoli. 2002. Revisión de las especies argentinas del género Haplopappus (Asteraceae, Astereae). Boletín de la Sociedad Argentina de Botanica 37: 115-133. 